\title{
Síndrome de West con aumento de inmunoglobulinas en líquido cefalorraquídeo
}

\author{
Sres. José Grass*, David Vantman V.*
}

\begin{abstract}
A study ou the period fronı a child's until his death, and whose mnther had got trichinosis at the 37 th week of gestition, was done. The child sutfered fom spasms called West's syndrome since his secord month of iile: A raise in immunoylobulins and positive results in some indirect semological tests related to trichinela spiralis were presented in the blood as well as in the cerebrospinal fluid.

The direct investigatiun to trace the presence of a larvae in the tissues didn't work out.

As the tiolugy of this syndrome it was pustulated an immuno-reactive phenomenon caused by parasitologicall antigens, which would have penetrated the placentury barrier. This statement should not be concluded as perentury.
\end{abstract}

El sindrome de West o Espasmos Infantiles es una fonna de epilepsia del tipo Pequeño Mal Motor, afecta a lactantes y consiste en crisis bruscas de espasmos en flexión de corta duración (1 a 4 seg.), que cursa generalmente en salvas y se caracteriza al E.E.G. por la hipsarritnia. Es un mal pronóstico dejando frecuentemente secuelas psíquicas y motoras importantes o evolucionando a formas más graves como el síndrome de LenoxGastaut. $1,2.3$

Su etiopatogenia es poco conocida, pero pueden intervenir en su desarrollo, con escasa frecuencia, encefalopatias de origen inteccioso. Es por esto que se analiza el caso clínico de un niño afectado por esta patología, cuya madre presentó infección por trichinella spiralis a las 37 semanas de gestación; este estudio se enfocó, desde el principio, en la búsqueda de un posible pasaje de larvas o de metabolitos de ellas desde la madre al feto, teniendo como inica referencia en la literatura a nuestro alcance un caso de triquinosis congénita que se presentó en un lactante de 6 semanas, cuya autopsia reveló la existencia de 22 quistes larvales por gramo de músculo. ${ }^{4}$

\section{CASOS CLINICOS}

De la madre: Paciente de 29 años de edad, cuyos ante-

\footnotetext{
"Alumnos de 7." año de Medicina de la Universidad de Chile,
} Sede Ocvidente, Hospibal San Juan de Dios. cedentes obstétricos indicaban 5 gestaciónes de las cuales tres dieron lugar a nacimientos de término y dos a abortos. A las treinta y siete semanas y media de amenorea, ingírió carne de cerdo, junto a su grupo familiar. A los 10 días, presentó un ciladro caracterizado por dolor periortitario, mialgias generalizadas, dolor en ambas Fosas renales y sensación febril; al ingreso, la paciente se encontraba febril, $\left(37.5{ }^{\circ} \mathrm{C}\right)$, taquicárdica, con edema bipalpebral, trismus, abdomen sensible sin visceromegalia y edema de extremidades. El hemograma revelo una leucocitosis de 9.800 y $18 \%$ de eosinófilos. La prueba de floculación con bentonita fue negativa, al igual que la prueba de precipitinas previa al parto, pero posterior a él, se positivizó la prueba de floculación con bentonita. La intradennorseacción de Bachman fue positiva. El resto del grupo familiar presentó un cuadro clírico y hemograma similares.

La evolución del embarazo hasta el momento de consulta estaba dentro de límites normales, de acuerdo con los parámetros de peso, altura uterina y latidos cardiofetales.

Para ver la influencia de la triquinosis sobre la uni. dad fetoplacentaria, se realizaron los siguientes exámenes: Amniocentesis que fue nomal para un niño de término, salvo la prueba de floculación con bentonita que fue sospechosa. La ecografta mostró un feto de 37 semanas con franca disminución de líquido amniótico y placenta con imágenes puntiformes sugerentes de calcificaciones basales e intercotiledonares, las cuales fueron posteriormente demostradas al examen macroscópico. Se practicó un test de reposo (RBNS) que resultó no reactivo con desaceleraciones tardías en relación a dinámica espontánea. Se indicó cesarea, durante la cual se encontrô escasa cantidad de líquido amniótico con meconio espeso. Se tomaron muestras de decidua, miome- 
trio y músculo recto anterior del abdomen, cuyas biopsias y triquinoscopias resultaron negativas. Con la mitad de la placenta, se efectuó triquinoscopía e inoculación experimental en animales, ambos negativos.

Clínica y estudio del niño: Se trata de un recién nacido de término adecuado a la edad gestacional, de sexo masculino, que presentó una asfixia neonatal moderada

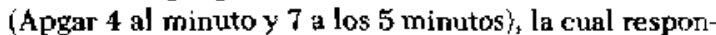
dio al tratamiento habitual y antes de las 48 horas se encontraba bien. Al mes y medio de vida, el niño presento un eritema marbiliforme de ambas extremidades inferiores, que pasó completamente en dos días, sin tratumiento. A los dos meses de vida fue hospitalizado por presentar un sindrome diarréico con deshidratación y un cuadro convulsivo de comienzo tocal y secundariarnente generalizado, acompañado de desviación de la mírada y seguido de hipertonía flexora de extremidades superiores y extensora en las inferiores, trismus y respiración algo irregular, con períodos cortos de apnea; este cuadro convulsivo se mantuvo con caracteres de status epil éptico durante dos dias cediendo con diazepam, para continuar posteriomente con crisis esporadicas, casi siempre focales y sin pérdida de conciencia. A su vez, presentó un aumento del tono muscular que cedió parcialmente con el uso de nitrozepam y corticoides.

El estudio de las pruebas serologicas para triquinosis en sangre de cordón, fue positiva para la floculación con bentunita y negativa para las precipitinas; a las 8 semanas, repitiendo estos exámenes, la floculación con bentonita se negativizó y se positivizó la otra. Se etectuó la intradernorneacción de Bachman cuyo resultado fue negativo.

El estudio seriado de inmunoglobulínas mostró una baja de Ig-M e Ig-C en sangre de cordón, pero a las 7 semanas tanto la Ig-M como la $\mathbf{~} \mathbf{g}-\mathbf{A}$ se encontraron elevadas (Cuadros $\mathrm{N},{ }^{\circ} 1,2$ y 3 ). Posteriomente a las 10 semanas, se encontró aumento en la lg-A e Ig-G, que se hicieron normales a las 20 semanas.

Se practicaron curvas de electroforesis de proteinas encontrándose hipogamaglobulinemia, que se mantivo en los controles posteriores.

Se efectuó un estudio completo de toxoplasmosis, enfermedad de Chagas, rubéola, lúes y citomegalovirus, todos los cuales fueron negativos. Durante la evolución del cuadro, uno de los hemagramas revelo anemia con anisocitosis, microcitosis, esferocitosis, poiquilocitosis,

\section{Cuadro 1}

\section{Cuantificación de IgA en suero}

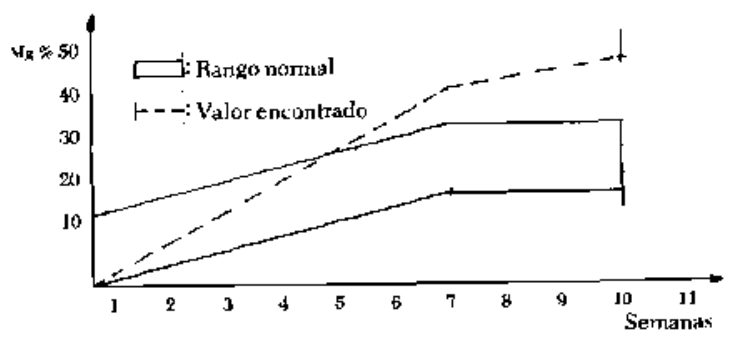

Cuadro 2

\section{Cuantificación de IgM en suero}

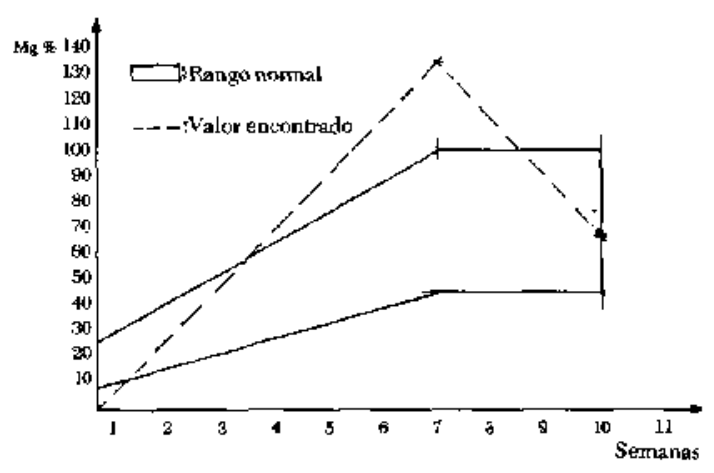

Cuadro 3

Cuantificación de IgG en suero

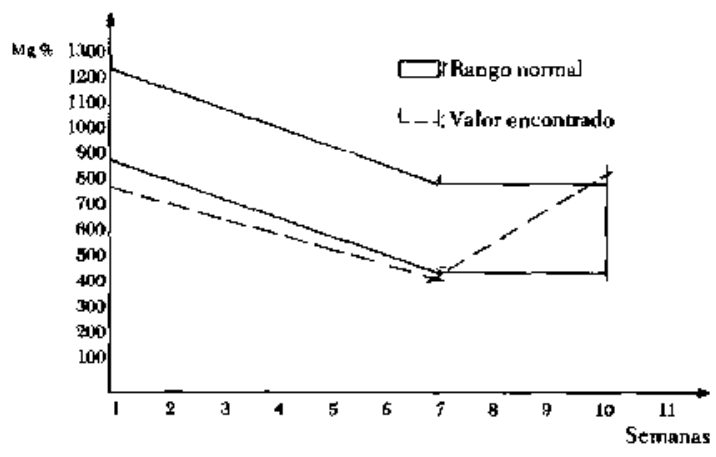

acantocitosis e hipocromia, que, unido a una elevación de las fracciones 1,2 y 3 de la deshidrogenasa lactica $y$ un cuadro febril de 2 dias, hicieron sospechar la existen. cia de una hemblisis breve, que no fue mayormente estudiada.

El estudio de enzimas de origen muscular como la transaminasa oxalacética y la fracción CK-MM de la creatinfosfoquinasa, mostró aumento.

Los primeros estudios de L.C.R. fueron nomales, salvo la mantenida positividad de la reacción de $\mathbf{P a n d y}$ $A$ los 70 días, el líquido era xantocrómico con aumento de proteínas, célulass y glutamina. Iloculación con ben. tonita positiva y ausencía de eosinófilos en el centrifugado. Posteriormente, se encontró gran aumento de IgA en el L.C.R., con un valor 10 veces superior al maximo aceptable, acompañado de una moderada elevación del complemento; ìrego de 30 días, la lg-A tenía un valor 3 a 4 veces sobre lo nomal (Cuadro 4).

Se efectuó determinación de Ig-M especifica para a]gunos antígenos de triłuinosis, tanto en L.C.R. como en suero, resultando ambos exámenes negativos.

Se practicaron, además, tres electroencefalogramas seriados que mostraron alteraciones compatibles con un sindrome de West; a su vez, se estudió el encéfalo por medio de tomografía axial computarizada que fue inforinada como normal. 
A los 7 meses de edad el nin̄o fallece de bronconeumonía, no encontrándose en la necropsia otro típo de lesiones, ni quistes parasitarios al examen histopatoló yico: se electuó rriquinoscopia e inoculación experimental de diatragma en animales, resultanda ambos negativos.

\section{Cuadro 4}

Cuantificación de IgA en L.C.R.

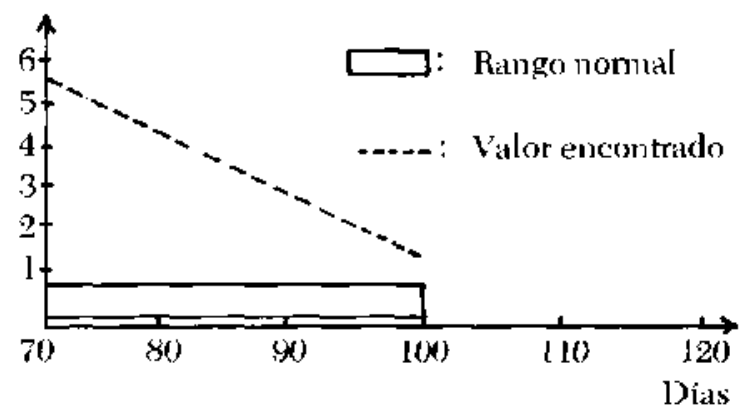

\section{DISCUSION}

Desde el punto de vista obstétrico, se puede pensar que la unidad fetoplacentaria no era normal. lo que se tradujo en la presencia de meconio en el líquido amniótico, oligoamnios y test de reposo catalogado como no reactivo. Basaindose en los antecedentes de 2 Fórceps y una cesárea anterior, se presenta como más probable que las alteraciornes encontradas en este caso, se deban a las mismas causas que deterninaron las intervenciones obstétricas anteriores, y quedaria como más remota la posibilidad de que se tratase de una placentitis u otra patologia ocasionada por la parasitosis de la madre.

A pesar de que no hubo hallazgo de T. spiralis en el lactante, existen hechos clínicos y de Jaboratorio que perniten sospechar la posibilidad de un traspaso de antígenos solubles o particulados, a través de la placenta, en cantidad suficiente como para poder estimular una respuesta inmune en el recién nacido. Esto sería apoyado por los siguientes hechos: 1. El hallazgo de una placenta con alteraciones morfológicas, lo que aumenta la posibilidad de "shunt" matemo-fetal, haciéndose la placenta parcialmente inefectiva en su función de barrera. 2. La positividad de las pruebas serológicas indirectas usadas habitualmente en la pescyuisa de antigenos de $T$. spiralis circulantes, todas ellas con muy bajo porcentaje de error ${ }^{5.6 .5}$ como son la floculación con bentonita sospechosa en el lícuido amniótico, positiva en la sangre de cordón umbilical y positiva en el líquido celaloraquídeo del lactante y la positividad de la prueba de las precipitinas en forma más tardía. La negatividad del estudio de Ig-G e Ig-M específicas para triquinosis en sangre y L.C.R. podrian ser atribuidos a que miden determinantes antigénicas diferentes a las pruebas serológicas anteriores y que pudieran estar ausentes o en concentraciones no detectables en este caso. Al mismo tiempo, la negatividad del test de Bachman pudiera ser atribuida a la misma causa. 3. La curva de inmunoglobulina, desde la etapa de recièn nacido hasta el momento de fallecer (Cuadros 1, 2 y 3), es compatible con unat respuesta a una noxa infecciosa ocurrida durante el ailtimo trimestre del embarazo, ${ }^{7.4}$ 4. 10. 11 que no sería ninguna de las infecciones congénitas habituales.

Considerando que la Ig-M estaba aumentada, no atraviesa la barrera placentaria y que esta alteración no se presentó en forma inmediata en el recién nacido, se podría explicar por el tiempo de latencia que requiere la formación de anticuerpos, unido a que el paso de antígenos debe haber ocurrido muy poco tiempo antes del término del embarazo. Debe considerarse gue en muchos casos de triquinosis comprobada, en que la infección es poco masiva, el aumento de anticuerpos se puede detectar después de los 60 días de exposición. ${ }^{12}$

El cuadro clínico presentado por el niño coresponde a un Sindrome de West, ${ }^{1,2,13}$ pero con la particularidad de cursar con un aumento de lg-A en el L.C... . no descrita antes por là literatura para ninguna otra patología, sea neurológica o no. ${ }^{\text {is. }}$ if. 17. $18 .{ }^{19}$ Este hecho, ademis de tener una tomografía axial computarizada encefálica normol ${ }^{13}$ y un estudio anătomo-patológico del encéfalo también normal, permite suponer una etiopatogenia diferente a las habituales, que en este caso pordría tratarse de un fenómeno immunorreaccional, cuyo organo más comprometido the el encéfalo. Esto puede ser sustentado por el hecho de que en las infecciones por T. Spiralis, parte de la clínica se debe a alteraciones de este tipo, como ocurre con la miocarditis triquinósica.

\section{AGRADECIMIENTOS}

- Dra. Milena Monari por la asesoria técnica prestada en el Hospital de Neurocirugía.

- Sra. Miriam Lorca por la colaboración dispensada. 
Damos nuestro más sincero reconocimiento $y$ agradecimiento a:

-Dr. Antonio Atías por haber corregido este trabajo.

- Dra. Elba Wu por la asesoria técnica.

\section{RESUMEN}

Se realizó un estudio desde el nacimiento hasta la muerte de un niño, cuya madre contrajo triquinosis a las 37 semanas de gestación. Desde el $2 .^{\circ}$ mes de vida presentó un cuadro convulsivo catalogado como sindrome de West, que cursó con elevación de inmunoglobulinas y positividad de algunas pruebas serológicas indirectas para trichinella spiralis, tanto en sangre como en L.C.R. Los estudios directos para buscar la larva en los tejidos resultaron negativos.

Se postuló como etiología de este síndrome un fenómeno inmuno-reaccional provocado por antigenos del parásito que habrian atravesado la barrera placentaria; lo que no se logsó concluir en forma definitiva.

\section{REFERENCLAS}

${ }^{1}$ Barsaquer Bordas, L. Neurología Fundamental, Cap. 13, pp. 463-465, tercera edición, 1976, Graficas Klimax, Espana.

${ }^{2}$ Gastaut, H.; Roger, J, Soularyol, R., et Pinsand, N. L'encefa lopathis myoclonijue infantile avec hipsarytmia. Encyclopedie Médico Chirurgicale, 17045 A 80, Masson et Cie edit., Pans, 1964.

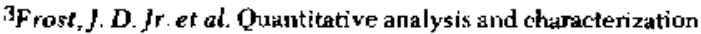
of intartile spasms. Epilepsia 19 (3): 273-82, 1978.

${ }^{4} B$ owns $T, K$. R. Discovery of Trichina Cists in the diafragm of a six weeks old child. J. Parast. 38: 367, 1952.

${ }^{5}$ Kagan, I, G., and Noman, $L$. In Trichinosis in men and animals. ed. S. E. Gould. Springfietd, IU: Charles C. Thomas, 222-268, 1970 .
6Schenone. H.: Knierim, F.; Concha, Li; Aranda, R.; Cofre, H. and Hojas, A. Investigactón de infecciones triquinósicas inaparentes mediante reacciones inmumobiológicas, Bol. Chileno Parasit. 24: 1 18, 1969.

7 Negro Ponzi et al. The inmune response of the fetus in the diagnasis of intrauterne infections. Ig-M and Ig-A concentration in the umbilical cord blood titers of rubella and cytomegalic virus antibodies. Boll. 15t Sieroter Milan 56 (2): 171-80, 1977.

8Furfora, M. et al. Ig-A and Ig-M inmunoglobulins in the umbilical cord blood. Minerua Peadiatr. 28 (5): 267-72, 1977.

Gandolfo Caramello, MT et al. Signs of fetal inmunology. Embryophathy caused by viruses: Deternination of Ig-M and Ig-A in the umbilical cord blood of newborn infants in relation of intrauterine infections. Control clinical examination of infants with high levels of Ig-M and $I g-A$ at birti. Boll Ist Sieroten Milan 56: (2): 181- 88 , 1977.

10Modzelewska-Kolaso, A. Determination of Ig-M and Ig-A in unbilical cord blood of newborm infants as a test in the early diagnosis of intrauterine intection. Pol Tyg Lek. 31 (51): 2169 $70,1976$.

I'Aggazzotti Zanasi, G. et al. Concentration of Ig-M and Ig-A in the umbilical cord blood of 400 newbom infants: possible significate and correlations with prenatal infections. G. Batteriol Virol Inmunol, 70 i $1-6)$; 49-59, 1977.

${ }^{12}$ Kagan, I. G. and Quist, K. D. In H.D. Srivastuva Commemoration Volume, Izatuagar, J. P., India: Div. of Parasit. Indias Vet. Res. Institute. pp. $271-277,1970$.

13 Gastout, H. et al. Computarized tomography in the study of West's syndrome. Dev. Med. Child Nerol. 20 (1): 21.27, 1978.

${ }_{14}$ Mikula, $T$. et al. Evaluation of snme serologic test for diagnosis of trichinosis. Bratisl Lek Listy 68 (1): 82-90. 1977.

${ }^{15} \mathrm{Link}, \mathrm{H}$. The value of cerebrospinal lluid inmunoglobulin analysis in clinical neurology. Hiv. Patol. Nerv. Ment 97 (6): 323-4. 1976.

${ }^{16}$ Mistens, $C_{+}$et al. Inmunoglobulin concentation in cerebrospinal tluid in children. Studies reparding age dependency and changes during inflamatory disease of CNS. Klin Peadiatr. 189 (2): 151-4, 1977.

${ }^{17}$ Lebos, l. et al. Electrophoretic study and deternination of inmunoglobulins in cerebrospinal tluid. Lille Med. 22 (1): $22-5,1977$.

lofederico, A. et al. Cerebrospinal fluid in neurological diseases. Hiv. Neurol. 46 (3): 183-93, 1976.

19 Cranrot, $K$. and Laurell, C. B. Measurement of Ig-G and albu* min content of cerebrospinal Đluid, and its interpretation. Clin Chem. 20: $571-3,1974$. 